\title{
Influencia de la suplementación durante el embarazo sobre el desarrollo cognitivo del niño
}

\author{
Influence of supplementation during pregnancy on the cognitive development of the child \\ Influência da suplementação durante a gravidez no desenvolvimento cognitivo da criança
}

\author{
Reina Marivel Iñiguez Jiménez \\ macainiguez@gmail.com \\ https://orcid.org/0000-0002-0376-9208
}

\author{
Stephanie Marie Cruz Pierard \\ smcp0893@gmail.com \\ https://orcid.org/0000-0003-4100-5898
}

\author{
Samuel Olegario Iñiguez Jiménez \\ iniguezjimenez@gmail.com \\ https://orcid.org/0000-0002-4722-7611
}

Pontificia Universidad Católica del Ecuador, Quito-Ecuador

Recibido 29 de marzo 2021 | Arbitrado y aceptado 16 de abril 2021 | Publicado en 4 de mayo 2021

\begin{abstract}
RESUMEN
Una nutrición adecuada durante el período prenatal es esencial para el desarrollo cerebral y la función cognitiva del recién nacido. Los estudios epidemiológicos han demostrado que la ingesta de folato, yodo y hierro durante la gestación repercute en el desarrollo cerebral del feto. Sin embargo, la relación con otros suplementos es menos clara. Objetivo. Analizar la evidencia científica disponible en lo relativo al efecto de la alimentación de la madre en el desarrollo cognitivo del niño. Método. Se realizó una búsqueda bibliográfica en Pubmed mediante términos Mesh, entre 2011 y 2021, en idioma español, inglés y francés, con bajo riesgo de sesgo. Se identificaron 116 investigaciones, tras excluir duplicados, protocolos y revisar la idoneidad de cada artículo mediante los criterios de selección, 10 estudios fueron incluidos en la presente revisión. Resultados. Entre los 10 artículos seleccionados la población total fue 2731 mujeres embarazadas, mayores de 18 años. Se identificó que la suplementación materna con al menos: 900 $\mathrm{mg} /$ día de colina mejora la memoria visual, la suplementación entre 400 a $600 \mathrm{mg} /$ día de DHA y 150 $\mathrm{mg} /$ día de EPA mejora la atención visual y la suplementación entre 400 a $600 \mu \mathrm{g} /$ día de ácido fólico beneficia el nivel cognitivo del niño. Conclusión. Se identificó que algunos componentes del complejo B como la colina, el ácido fólico y determinados ácidos grasos poliinsaturados como el docosahexaenoico y el eicosapentaenoico, pueden tener un efecto positivo en el desarrollo cognitivo del niño siempre y cuando se suplementen adecuadamente según los requerimientos de la gestante.
\end{abstract}

Palabras clave: Desarrollo cognitivo; dieta; embarazo; niño; suplementación RI: Licenciada en Nutrición Humana, Pontificia
Universidad Católica del Ecuador. Master Oficial en Nutrición Humana, Universidad de Granada. Nutricionista en Bonne vie. Pontificia Universidad Católica del Ecuador, Ecuador. SC: Licenciada en Nutrición Humana PUCE. Maestría Internacional en Nutrición Infantil ESNECA Business School Virtual en convenio con Universidad Católica del Cayo Argentina. Diplomado de Especialización Profesional en
Nutrición Clínica IIDENUT. Diplomado de Nutrición Clínica IIDENUT. Diplomado de nutrición y dietoterapia en ITSEI. Nutricionista en Bonne vie / Paradise One Medicina Genética / PSP Sanofi. Pontificia Universidad Católica del Ecuador, Ecuador.

SI: Licenciado en Psicología, Universidad Técnica Particular de Loja. Licenciado en Fisioterapia, Pontificia Universidad Católica del manual ortopédica en el tratamiento del dolor Universidad Europea de Madrid. Docente Universidad Europea de Madrid. Docente Católica del Ecuador, Ecuador.

\begin{abstract}
An adequate maternal nutrition during the prenatal period is essential for the brain development and cognitive function of the new-born. Epidemiological studies have shown that the intake of folate, iodine, and iron during pregnancy affects the development of the fetal brain. However, the relationship with other supplements is still scarce. Objective. To analyze the available scientific evidence of the effects of maternal diet-during pregnancy the cognitive development of the child. Methods. a search for studies was carried out in PubMed using Mesh terms, published from 2011 to 2021, in Spanish, English, and French, with a low risk of bias. 116 investigations were identified, after excluding duplicates, protocols and reviews, a total of 10 studies were used for the present review. Results. In the 10 selected articles, the total population was 2731 pregnant women, older than 18 years. Maternal supplementation with at least: $900 \mathrm{mg} /$ day of choline improved visual memory, a supplementation with at least 400 to 600 $\mathrm{mg} /$ day of DHA, $150 \mathrm{mg} /$ day of EPA improves visual attention, 400 to $600 \mathrm{ug} /$ day of folic acid exerted a benefit in the cognitive level, of the child. Conclusion. It was identified that some components of the B complex such as choline, folic acid, and specific polyunsaturated fatty acids such as docosahexaenoic and eicosapentaenoic, can have a positive effect on the cognitive development of the child as long as they are adequately supplemented according to the requirements of the pregnant woman.
\end{abstract}

Key words: Cognitive development; child; diet; pregnancy; supplementation 
RI: Licenciada en Nutrición Humana, Pontificia Universidad Católica del Ecuador. Master Oficial en Nutrición Humana, Universidad de Granada. Universidad Cáĺlic del Ecuedor, Ecuador.

SC: Licenciada en Nutrición Humana PUCE Maestría Internacional en Nutrición Infantil ESNECA Business School Virtual en convenio con Universidad Católica del Cayo Argentina. Nutrición Clínica Nutrición Deportiva UDLA. Docente de nutrición y dietoterapia en ITSEI. Nutricionista en Bonne vie / Paradise One Medicina Genétic / PSP Sanofi. Pontificia Universidad Católica de Ecuador, Ecuador.

SI: Licenciado en Psicología, Universidad Técnica Particular de Loja. Licenciado en Fisioterapia, Pontificia Universidad Católica del Ecuador. Máster universitario en terapia manual ortopédica en el tratamiento del dolor Universidad Europea de Madrid. Docente universitario PUCE. Pontificia Universidad Católica del Ecuador, Ecuador.

\section{RESUMO}

A nutrição adequada durante o pré-natal é essencial para o desenvolvimento do cérebro e função cognitiva do recém-nascido. Estudos epidemiológicos demonstraram que a ingestão de folato, iodo e ferro durante a gravidez afeta o desenvolvimento do cérebro do feto. No entanto, a relação com outros suplementos é menos clara. Objetivo. Analisar as evidências científicas disponíveis sobre o efeito da dieta da mãe no desenvolvimento cognitivo da criança. Método. Foi realizada pesquisa bibliográfica no Pubmed com termos Mesh, entre 2011 e 2021, em espanhol, inglês e francês, com baixo risco de viés. Foram identificadas 116 investigações, após exclusão de duplicatas, protocolos e revisão da adequação de cada artigo utilizando os critérios de seleção, 10 estudos foram incluídos na presente revisão. Resultados. Entre os 10 artigos selecionados, a população total foi de 2.731 gestantes, maiores de 18 anos. Foi identificado que a suplementação materna com pelo menos: $900 \mathrm{mg} / \mathrm{dia}$ de colina melhora a memória visual, a suplementação entre 400 a $600 \mathrm{mg} /$ dia de DHA e 150 mg / dia de EPA melhora a atenção visual e a suplementação entre 400 a $600 \mu \mathrm{g}$ / dia de o ácido fólico beneficia o nível cognitivo da criança. Conclusão. identificou-se que alguns componentes do complexo B, como colina, ácido fólico, e certos ácidos graxos poliinsaturados como docosahexaenóico e eicosapentaenóico, podem ter efeito positivo no desenvolvimento cognitivo da criança, desde que devidamente suplementados de acordo com os requisitos da mulher grávida.

Palavras-chave: Desenvolvimento cognitivo; dieta; gravidez; menino; suplementação

\section{INTRODUCCIÓN}

$\mathrm{L}$ a gestación es una de las etapas de mayor relevancia en la vida de la mujer, comprende un período aproximado de 9 meses que conlleva diversos cambios tanto anatómicos como fisiológicos en el organismo (1). Durante este tiempo, el feto se desarrolla en el vientre materno a través de diferentes mecanismos que proporciona lo necesario para cumplir con los requerimientos del crecimiento y nutrición al nuevo ser $(1,2)$.

Para lograr una adecuada evolución del embarazo, la madre debe pasar por distintas adaptaciones metabólicas y fisiológicas como una ganancia de peso apropiada, cambios en la composición corporal, incremento en el gasto cardíaco, alteraciones en la piel y modificaciones en diversos sistemas como el nervioso, urinario, digestivo y respiratorio para albergar a la vida en formación (3). Estas adecuaciones y el desarrollo del feto implican necesidades alimentarias incrementadas de energía, pero sobre todo de nutrientes específicos (3).

En este contexto, el embarazo representa un lapso de vulnerabilidad biológica para la gestante, por ende, la nutrición es una parte prioritaria tanto en el adecuado desarrollo de la vida fetal como en la salud física de la madre, para mantener en ambos un buen estado nutricional y posteriormente respaldar la lactancia $(4,5)$.

La nutrición en el embarazo debe ser completa, equilibrada y suficiente. En cuanto a la cantidad de calorías (kcal) necesarias, se recomienda un incremento de 300 a $500 \mathrm{kcal}$ por día en el segundo y tercer trimestre para lograr la ganancia de peso esperada que varía entre 5 a 18 kilogramos dependiendo del estado nutricional inicial de la madre (Tabla 1) (68). 
Tabla 1. Recomendaciones de ganancia de peso esperada según el índice de masa corporal pregestacional.

\begin{tabular}{ccc}
\hline & IMC pregestacional $\left(\mathbf{k g} / \mathbf{m}^{2}\right)$ & Recomendación $(\mathbf{k g})$ \\
\hline Bajo peso & $<18,5$ & $12,7-18,2$ \\
Peso normal & $18,5-24,9$ & $11,4-15,9$ \\
Sobrepeso & $25-29,9$ & $6,8-11,4$ \\
Obesidad & $>/=30$ & $5,0-9,1$ \\
\hline
\end{tabular}

IMC, índice de masa corporal

Con respecto a los macronutrientes, la distribución recomendada en carbohidratos es del 50 a $55 \%$ de las calorías totales o una ingesta de $175 \mathrm{~g}$ diarios (mejor de fuentes integrales), proteínas del 15 a $20 \%(1,1 \mathrm{~g} / \mathrm{kg} /$ día $)$ y con respecto a las grasas entre 30 a $35 \%$ del valor calórico $(20$ a $35 \mathrm{~g}$ al día preferiblemente de fuentes insaturadas) $(7,8)$. En la Tabla 2 se puede apreciar las recomendaciones de energía y macronutrientes elaborada a partir de datos del Institute of Medicine (9) y de otras investigaciones (10-12).

Tabla 2. Recomendaciones de energía y macronutrientes en el embarazo

\begin{tabular}{|c|c|}
\hline Nutriente/Energía & Recomendación \\
\hline Energía & $\begin{array}{l}\text { 10 Trimestre + } 0 \mathrm{kcal} / \text { día } \\
2^{\text {o }} \text { Trimestre }+340 \mathrm{kcal} / \text { día } \\
3^{\text {o }} \text { Trimestre }+452 \mathrm{kcal} / \text { día }^{2}\end{array}$ \\
\hline Proteínas & 60 g/día (1,1 g/kg peso/día) \\
\hline Hidratos de carbono & 45-65\% energía ( $\geq 175$ g/día) \\
\hline Grasas & $20-35 \%$ de la energía (similar a no embarazada) \\
\hline Ingesta de líquidos & 1,5-2,3 l/día \\
\hline
\end{tabular}

A su vez, en el embarazo se produce un aumento de requerimientos en algunos nutrientes, por lo que es importante escoger bien los alimentos a consumir en la dieta de la gestante para prevenir futuras complicaciones en el feto (5). Este es el caso de los micronutrientes, durante el embarazo se incrementan todos los requerimientos de vitaminas y minerales, siendo más importante centrarse en aquellos representados en la Tabla 3, la cual está basada en la Guía de Alimentación para Embarazadas de Sánchez (13). 
Tabla 3. Micronutrientes importantes en el embarazo

\begin{tabular}{|c|c|c|c|}
\hline MICRONUTRIENTE & FUNCIÓN & $\begin{array}{c}\text { DÓNDE SE } \\
\text { ENCUENTRA }\end{array}$ & $\begin{array}{c}\text { AUMENTO } \\
\text { RECOMENDADO }\end{array}$ \\
\hline \multicolumn{4}{|c|}{ VITAMINAS } \\
\hline Ácido Fólico & $\begin{array}{l}\text { Prevención de } \\
\text { defectos del tubo } \\
\text { neural y parto } \\
\text { prematuro. }\end{array}$ & $\begin{array}{l}\text { Verduras y hortalizas } \\
\text { verdes, legumbres, } \\
\text { frutas, frutos secos y } \\
\text { cereales integrales. }\end{array}$ & $+200 \mu \mathrm{g}$ \\
\hline Vitamina D & $\begin{array}{c}\text { Aumento de absorción } \\
\text { del calcio. }\end{array}$ & $\begin{array}{c}\text { Pescados azules, } \\
\text { lácteos no desnatados } \\
\text { y sol. }\end{array}$ & $\begin{array}{c}\text { Mantener consumo } \\
(5 \mu \mathrm{g})\end{array}$ \\
\hline Vitamina B6 & $\begin{array}{c}\text { Desarrollo del sistema } \\
\text { nervioso y } \\
\text { crecimiento del feto. }\end{array}$ & $\begin{array}{l}\text { Carnes, pescados, } \\
\text { frutos secos y } \\
\text { legumbres. }\end{array}$ & $+0,6 \mu \mathrm{g}$ \\
\hline Vitamina B12 & $\begin{array}{l}\text { Formación de la } \\
\text { espina bífida del feto. }\end{array}$ & $\begin{array}{l}\text { Alimentos de origen } \\
\text { animal. }\end{array}$ & $+0,2 \mu \mathrm{g}$ \\
\hline Vitamina C & $\begin{array}{l}\text { Crecimiento y } \\
\text { reparación de tejidos } \\
\text { del feto. Mejora } \\
\text { absorción de hierro y } \\
\text { sistema inmunitario } \\
\text { de la madre. }\end{array}$ & $\begin{array}{l}\text { Frutas y verduras } \\
\text { (frescas y crudas). }\end{array}$ & $+10 \mathrm{mg}$ \\
\hline \multicolumn{4}{|c|}{ MINERALES } \\
\hline Hierro & $\begin{array}{c}\text { Aumenta el volumen } \\
\text { sanguíneo de la } \\
\text { madre. }\end{array}$ & $\begin{array}{c}\text { Carnes (vísceras, } \\
\text { conejo, liebre, ternera } \\
\text { y buey) y mariscos. }\end{array}$ & $+9 \mathrm{mg}$ \\
\hline Calcio & $\begin{array}{l}\text { Aumenta la densidad } \\
\text { ósea materna y fetal. }\end{array}$ & $\begin{array}{l}\text { Lácteos, verduras, } \\
\text { hortalizas y } \\
\text { legumbres. }\end{array}$ & $+200 \mathrm{mg}$ \\
\hline Yodo & $\begin{array}{c}\text { Síntesis de hormonas } \\
\text { tiroideas necesarias } \\
\text { para el crecimiento } \\
\text { del feto y maduración } \\
\text { de tejidos. }\end{array}$ & $\begin{array}{l}\text { Pescados, mariscos, } \\
\text { algas y sal yodada. }\end{array}$ & $+25 \mathrm{mg}$ \\
\hline
\end{tabular}


El crecimiento y desarrollo del feto tiene una duración de 38 a 40 semanas de gestación, en el primer trimestre (semana 1 a la 12) se produce la concepción, implantación y formación del embrión y la placenta, que proveerá los nutrientes y oxígeno, desde la semana 5 empieza el desarrollo de todos los principales sistemas y estructuras del bebé (cerebro, corazón, médula espinal, tracto gastrointestinal, etc.). También en este período se presenta mayor riesgo de daños a causa de factores que pueden causar anomalías congénitas $(2,14)$.

Durante el segundo trimestre (semana 13 a la 28), continúa el crecimiento y progreso de todas las estructuras orgánicas, aparecen los genitales y brotes dentarios, así como pequeños movimientos fetales que pueden ser percibidos por la madre. Finalmente, en el tercer trimestre (semana 29 a la 40), el cerebro crece rápidamente y el sistema nervioso es capaz de controlar algunas funciones del cuerpo, termina la maduración de todos los órganos y el bebé almacena hierro, calcio, fósforo y grasa, ganando el peso adecuado para nacer (14).

Cabe recalcar un aspecto de suma importancia, como es el desarrollo cognitivo fetal, dado que, para un óptimo desarrollo del mismo, se requiere del aporte adecuado de nutrientes principalmente en la etapa de la gestación y la infancia. Se conoce que los primeros años de vida son las etapas más activas del perfeccionamiento del cerebro. A esta edad, los niños están en pleno crecimiento y aprendizaje, por ende, aumentan los momentos de desarrollo cerebral para el funcionamiento cognitivo, entre la asociación de la madurez motora y el saber (15). Según Black (16), el tiempo comprendido desde la concepción hasta los 2 años del niño conlleva un rápido desarrollo cerebral y la nutrición juega un papel primordial, puesto que la cronicidad y gravedad de los déficits nutricionales han mostrado efectos diferenciales en el desarrollo del cerebro y en los procesos cognitivos y emocionales posteriores.

En varias investigaciones, se evidencia la relación entre la nutrición de la madre y el desarrollo cerebral del bebé. Por ejemplo, Rombaldi et al. (17), Hurtado (18) y López (19) consideran que un aporte adecuado de ácidos grasos poliinsaturados (omega 3) puede condicionar de manera positiva el desarrollo cognitivo o neurológico del feto. De igual forma, en una revisión sistemática (20) se determinó que embarazadas con bajos niveles séricos de vitamina $\mathrm{D}(<50 \mathrm{nmol} / \mathrm{L})$ tuvieron hijos con peor desarrollo mental, motor y del lenguaje en comparación con madres con ingestas adecuadas.

Además, Rogne et al. (21) mencionan que el consumo de vitamina B12 es necesario para un correcto desarrollo fetal y que niveles bajos de la misma se han asociado con impactos negativos a nivel cognitivo, motor y de crecimiento, lo cual se ha evidenciado principalmente en gestantes vegetarianas, veganas o que presentan enfermedad celiaca. Por otro lado, en una revisión sistemática realizada por Taylor et al., (22) encontraron que la suplementación con múltiples micronutrientes durante el embarazo influyó positivamente en la atención de los bebés 15 días después del parto. Sin embargo, como conclusión general se determinó que las intervenciones nutricionales durante el embarazo no necesariamente presentan un impacto significativo en los resultados cognitivos del niño, comprobándose la necesidad de mayor investigación al respecto. 
En España, estudios como el de Hurtado (18), López (19) y Englund-Ögge et al. (23) han colaborado con la literatura respecto a este tema. No obstante, sería recomendable la realización de más investigaciones para complementar los conocimientos en este aspecto relevante del embarazo y la nutrición.

Finalmente, con los antecedentes presentados, se puede verificar que existen asociaciones entre una alimentación materna adecuada durante la gestación, que aporte la cantidad y calidad suficiente tanto de macro como de micronutrientes, $y$ el correcto crecimiento y desarrollo del feto, especialmente a nivel cognitivo. Sin embargo, es imperativo recalcar la importancia de incrementar los estudios referentes a este tema ya que todavía no hay suficiente evidencia científica contundente para confirmar la relación de estas variables.

\section{MÉTODO}

S e realizó una revisión, siguiendo la metodología PRISMA (24). Se desarrolló una cadena de búsqueda extensa para no excluir literatura relevante en PubMed y se adaptó con términos MeSH la siguiente estrategia de búsqueda: (Pregnan*) AND ((Cognit*) OR (neurodevelopment) OR (intelligen*) OR (memory) OR (attention) OR (language)) AND ((Infant*) OR (child*)) AND ((Food*) OR (nutrition) OR (supplement) OR (diet) OR (dietary supplement*) OR (nutrient supplement)). Fueron aplicados los siguientes filtros: resumen, texto completo gratuito, texto completo, ensayo clínico, ensayo controlado aleatorizado, en los últimos 10 años, humanos, inglés, francés, español, femenino.

Se plantearon los siguientes criterios de selección basados en la importancia de la calidad de la dieta materna durante el embarazo en el desarrollo cognitivo del niño, indicados en metaanálisis previos $(1,22)$.

Los criterios de inclusión fueron:

- Gestantes en edades comprendidas entre los 18 y 45 años.

- Mujeres embarazadas sanas, sin patologías asociadas.

- Artículos publicados desde el 2011 hasta el 2021.

- Investigaciones en idioma castellano, inglés o francés con bajo riesgo de sesgo.

- Estudios transversales, prospectivos o retrospectivos

Los criterios de exclusión:

- Mujeres que declararon tomar fármacos que pudieran influir en su alimentación durante la gestación.

- Mujeres que recibieron suplementación durante el periodo de lactancia.

- Revisiones sistemáticas, cartas al editor y comunicaciones breves.

Para evitar riesgo de sesgo se realizó una búsqueda bibliográfica de la evidencia, siguiendo los criterios de inclusión y exclusión descritos. El protocolo de revisión se desarrolló utilizando el Manual Cochrane para Revisiones Sistemáticas de Intervenciones (25). 
De esta manera, se identificaron 116 investigaciones a través de la búsqueda inicial, al excluir duplicados, protocolos y revisar la idoneidad de cada artículo mediante los criterios de selección quedaron 10 estudios elegibles que se incluyeron en los resultados (Figura 1).
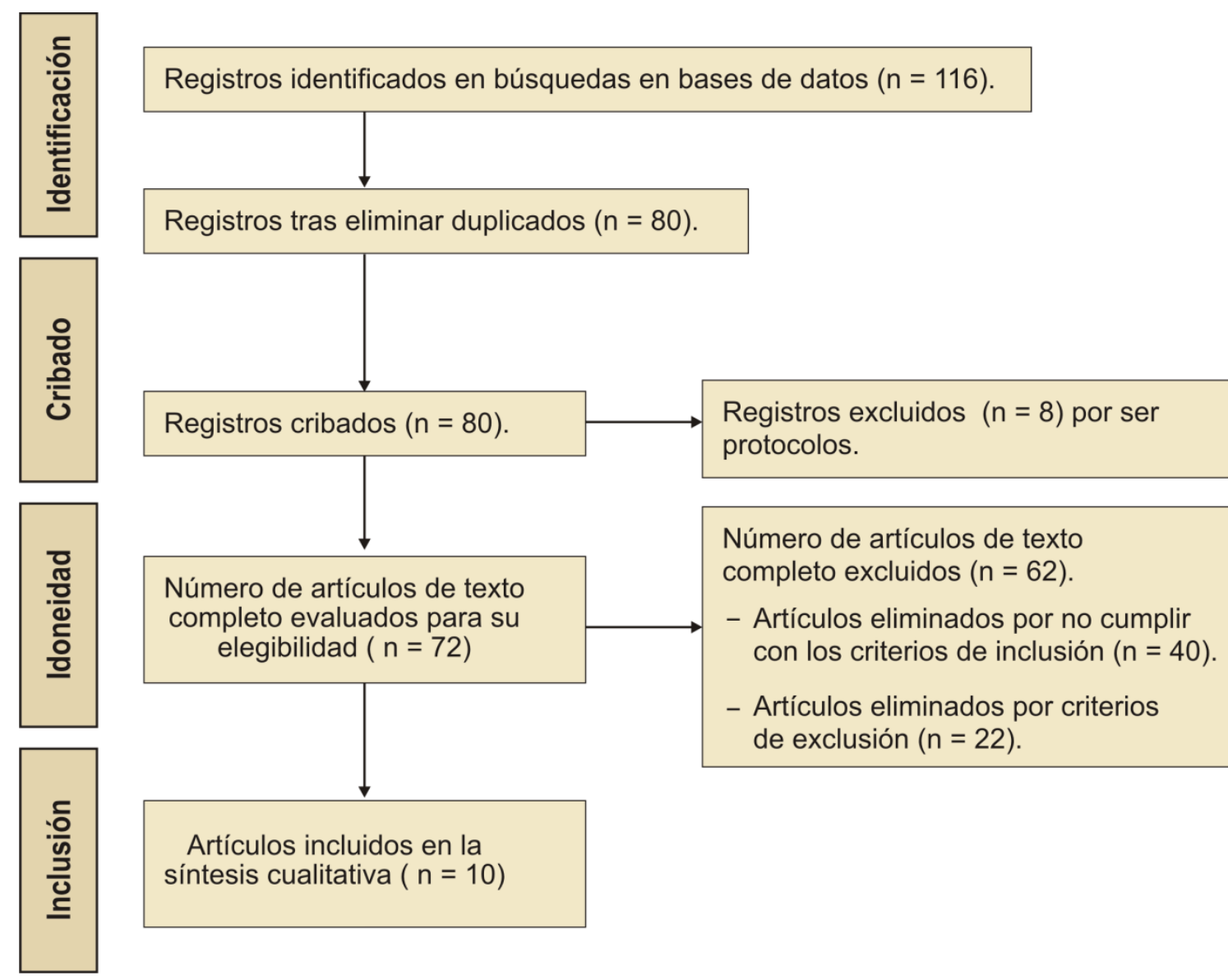

Artículos incluidos en la síntesis cualitativa $(n=10)$

Figura 1. Diagrama de flujo de la búsqueda sistemática.

\section{DESARROLLO Y DISCUSIÓN}

$\mathrm{U}$ n total de 10 artículos científicos se incluyeron en el presente trabajo de revisión acerca de la influencia de la alimentación de la madre durante la gestación sobre el desarrollo cognitivo del niño. En cuanto al diseño de los estudios, 9 fueron ensayos clínicos aleatorizados doble ciego (26-34) y 1 estudio (35) fue observacional.
La población total de las investigaciones analizadas fue de 2731 mujeres embarazadas mayores de 18 años.

Respecto a la intervención, los estudios tenían como base la dieta de la madre más la suplementación (colina, DHA/EPA, ácido fólico) en un periodo determinado del embarazo.

En este aspecto, se registraron 7 estudios con suplementación de DHA/EPA (28-35), de los cuales 4 tuvieron un efecto 
positivo, mejorando así el desarrollo cognitivo del niño. Sin embargo, en 3 estudios (32-34) no se evidenció tal efecto.

Un estudio con suplementación de DHA y ácido fólico (30) y un estudio con suplementación exclusiva de ácido fólico (27), también presentaron efectos positivos en el desarrollo cognitivo del niño, al igual que con la suplementación de colina en 1 estudio (26). La dosis óptima utilizada y los resultados de cada estudio se encuentran detallados en la Tabla 4.

Por otra parte, los instrumentos utilizados para la evaluación de la alimentación de las madres fueron historia dietética o cuestionario de frecuencia de consumo de alimentos (35). En cuanto a la medición del desarrollo cognitivo en los niños se manejaron diferentes instrumentos para su análisis, entre ellos la escala de inteligencia $(27,28)$, pruebas de desempeño continuo para niños (29) y pruebas para medir la velocidad de procesar información $(30,32)$, además, se emplearon métodos como pruebas de desarrollo de integración visual-motora (34), precisión de la localización del juguete (33), así como el comportamiento de la duración máxima de la mirada y el número de miradas a la habituación $(26,31,32)$.

Tabla 4. Características de los artículos incluidos en la revisión

\begin{tabular}{lll}
\hline $\begin{array}{c}\text { Suplemento y } \\
\text { dosis óptima }\end{array}$ & Resultados & Conclusiones \\
\hline
\end{tabular}

Colina

$900 \mathrm{mg} /$ día

Caudill et al. (26)

Ácido fólico 400 a 600 $\mu \mathrm{g} / \mathrm{dí} \mathbf{a}$

McNulty et al. (27) Azaryah et al. (30)

DHA

400 a 600 mg/día
Los bebés de las madres suplementadas con colina reaccionaron consistentemente más rápido en la velocidad de reconocimiento de imágenes, lo que indica mayor velocidad de procesamiento de la información (26).

Los niños nacidos de madres suplementadas con ácido fólico obtuvieron puntuaciones significativamente más altas en razonamiento de palabras (27).

Los niños del grupo suplementado con ácido fólico mostraron una fuerte conectividad funcional en el modo predeterminado, las redes sensoriomotoras y la red frontoparietal (30).

Se determinó mejor desempeño de los niños del grupo que sus madres fueron suplementadas con aceite de pescado en la prueba de reconocimiento de objetos (memoria) (30).
La suplementación con colina de la dieta entre las mujeres desde su tercer trimestre de embarazo, mejoró la velocidad de procesamiento infantil en relación con el funcionamiento cognitivo (26).

La administración continua de suplementos de ácido fólico a las madres durante el segundo y tercer trimestre del embarazo puede influir en el rendimiento cognitivo de sus hijos hasta los 7 años de edad (27).

El consumo de aceite de pescado durante el embarazo puede moldear el funcionamiento de la red en estado de reposo de los niños y producir efectos en el procesamiento cognitivo (30). 


\begin{tabular}{lll}
\hline $\begin{array}{c}\text { Suplemento y } \\
\text { dosis óptima }\end{array}$ & Resultados & Conclusiones \\
\hline
\end{tabular}

EPA

150 mg/día

Azaryah et al.

Colombo et al.

(31)

Colombo et al.

Gould et al. (33)

Meldrum et al.

(34)

Mulder et al. (32)

Ramakrishnan et

al. (29)

Rees et al. (35)
Los bebés de madres suplementadas con DHA mantuvieron altos niveles de atención sostenida durante su primer año. También se mostró una reducción significativa del desgaste en las tareas de habituación, especialmente a los 6 y 9 meses (31).

Las concentraciones de DHA en sangre materna en el momento de la inscripción se relacionó con el vocabulario productivo a los 18 meses (28).

En las pruebas de desempeño continuo para medir la atención, la descendencia en el grupo de DHA mostró una mejora en las puntuaciones (29).

En la prueba de agudeza visual de 9 meses, los bebés cuyas madres estaban en el grupo de DHA medio se desempeñaron significativamente mejor que aquellos con madres en los grupos de DHA bajo o alto (35).
El DHA prenatal puede afectar positivamente la atención y la regulación del estado de los bebés (31).

La suplementación prenatal con DHA mejoró la atención visual en la infancia, no se observaron beneficios consistentes a largo plazo en la niñez (28).

La exposición prenatal al DHA puede contribuir a mejorar la atención sostenida en los niños en edad preescolar (29).

La ingesta de DHA en el tercer trimestre específicamente, influye en el desarrollo de la agudeza visual hacia el final del primer año posnatal (35). 


\section{Discusión}

La alimentación de la madre durante el embarazo y cómo influye en diferentes aspectos de la vida del niño, entre ellos su desarrollo a nivel cognitivo, es un aspecto importante, aunque relativamente difícil de analizar, debido a que existen variaciones en cuanto a la dieta que cada embarazada opta seguir, así como los múltiples suplementos disponibles en el mercado para ayudar a alcanzar una nutrición adecuada tanto de la madre como el feto (22). Sin embargo, se ha logrado evaluar los efectos que causan determinados nutrientes gracias a estudios experimentales, con los que se han sentado las bases de la relación entre la dieta materna durante la gestación y el desarrollo tanto físico como neurológico del niño.

El objetivo de la presente revisión fue analizar la evidencia disponible sobre el efecto de la alimentación de la madre durante la gestación en el desarrollo cognitivo del niño. De los estudios examinados se pudo deducir que en el 70 $\%$ de los mismos se demuestra que la suplementación de la dieta materna con nutrientes como DHA, colina y ácido fólico en determinadas etapas del embarazo presenta un efecto positivo en el desarrollo cognitivo del niño a distintas edades, lo cual coincide con los resultados de la revisión sistemática y metaanálisis de Borge et al. (1) en la que se determinó que una mejor calidad de dieta materna presenta una asociación positiva con el desarrollo neurológico infantil.

En este aspecto, los nutrientes mencionados presentan un papel relevante en procesos clave del neurodesarrollo del niño durante la gestación; la colina participa en la señalización transmembrana durante la neurogénesis $y$ es precursora del neurotransmisor acetilcolina que posteriormente intervendrá en el proceso de aprendizaje y consolidación de recuerdos, los ácidos grasos como el DHA son necesarios para sintetizar fosfolípidos de membrana requeridos en la formación neuronal y el ácido fólico influye en la formación de la placa y tubo neural que luego se convierten en el cerebro y la médula espinal (36), por lo que se fundamenta que un adecuado aporte nutricional de los mismos mejora la ejecución de sus funciones.

Con respecto a la suplementación de colina, Caudill et al. (26) determinaron que en los bebés a la edad de 4 a 13 meses mejoró el funcionamiento cognitivo aumentando la velocidad de procesamiento de información, lo cual resalta la importancia de incluir este nutriente dentro de la dieta materna, que a pesar de estar presente en varios alimentos como carnes, legumbres y de sintetizarse en pocas cantidades en el organismo, menos del $10 \%$ de embarazadas logra cumplir con las recomendaciones diarias de $450 \mathrm{mg} /$ día $(37,38)$.

Se ha determinado diversos efectos de la ingesta materna de colina durante el embarazo en estudios tanto en animales como en humanos, entre ellos la programación epigenética de la salud posnatal, función placentaria, metabolismo energético, neurodesarrollo y función cognitiva infantil (38), por lo que se debería tomar en cuenta a este micronutriente dentro de los suplementos que normalmente se recomiendan a las mujeres embarazadas como el ácido fólico y otras vitaminas $\mathrm{y}$ minerales.

De la misma manera, varios estudios corroboran que la suplementación con al menos $900 \mathrm{mg} /$ día de colina en la dieta materna tiene un efecto positivo en el desarrollo cognitivo de la descendencia en 
cuanto a memoria visual $(39,40)$ y atención $(41,42)$.

Por otra parte, la suplementación de la gestante con 400 a $600 \mathrm{mg} /$ día de ácidos grasos poliinsaturados como el DHA y 150 $\mathrm{mg} /$ día de EPA, también mostró efectos positivos en cuanto a atención visual (28), atención sostenida (29), y regulación del estado de los bebes (31), así como en el desarrollo de la agudeza visual (35) y reconocimiento de objetos (30), principalmente en los primeros años de vida. El DHA constituye un componente imprescindible en la estructura de la retina (80 \%) y el cerebro humano (60\%), cumpliendo diversas funciones en este último como la regulación de la expresión génica, neurotransmisión y señalización celular, durante el desarrollo fetal e infantil las membranas de estos órganos se enriquecen con DHA lo que puede explicar los beneficios de su suplementación citados anteriormente (43).

Conviene enfatizar que la ingesta recomendada de EPA y DHA en mujeres embarazadas es de 200-375 mg/día (43), y según varias investigaciones el $95 \%$ de embarazadas no cumplen con estos requerimientos diarios, pudiendo deberse a que es necesario aumentar el consumo semanal de pescados y mariscos entre 224 a 336 gramos, o a su vez la utilización de suplementos para poder alcanzar estos valores, lo cual implica un encarecimiento de la dieta materna por lo que en muchos casos esta no se puede efectuar por problemas socioeconómicos de la gestante (44).

En otros estudios analizados en los que se suplementó a las madres con 800 mg (33) o 2,2 g/día de DHA (34) se llegó a la conclusión que este nutriente no influye en la cognición de los niños a los 2 o 12 años, pudiendo deberse al reducido tamaño de las muestras analizadas, ya que en otras investigaciones con dosis similares de suplementación pero con poblaciones más amplias se llegó a resultados opuestos (45-47). Asimismo, Meldrum et al. (34) sugieren que el efecto de la suplementación materna que se observa en los lactantes puede verse diluido por la dieta de los niños y otros factores ambientales, por lo cual no persisten en el tiempo y no se evidencian en edades superiores, esto coincide con lo mencionado por Colombo et al. (28) quienes indican que no se observan beneficios de la suplementación materna con DHA consistentes a largo plazo en la niñez.

Cabe recalcar que la diversidad de los estudios en cuanto a cantidades suplementadas de nutrientes y edad de la población evaluada también genera que los resultados sean muchas veces contradictorios. Por este motivo, para futuras investigaciones se recomienda analizar artículos en poblaciones con edades y niveles de suplementación similares para verificar hasta qué punto el efecto obtenido sigue siendo tangible $\mathrm{y}$ efectivo.

Otro de los nutrientes analizados fue el ácido fólico (vitamina B9), cuya suplementación con al menos $400 \mu \mathrm{g} /$ día mostró también beneficios a nivel cognitivo de los niños como fuerte conectividad funcional en el modo predeterminado (30), mejor razonamiento de palabras y cognición (27). La recomendación diaria de $\mathrm{B} 9$ en mujeres embarazadas es de 400 a $600 \mu \mathrm{g} /$ día, lo que también constituye un reto nutricional que en la mayoría de gestantes se cubre gracias a la suplementación (48).

El efecto positivo causado por el ácido fólico se explica por ser un nutriente clave 
en cuanto a la formación y desarrollo del cerebro, ya que interviene en procesos como la proliferación y crecimiento de las neuronas y la síntesis de neurotransmisores (27), además múltiples estudios coinciden en que la suplementación materna de folatos mejora el rendimiento cognitivo, especialmente en las habilidades verbales de los niños $(49,50)$.

Finalmente, cabe mencionar que la etapa de gestación es un desafío desde el punto de vista nutricional, por las necesidades incrementadas de nutrientes y las limitaciones que se pueden dar por parte de la madre a cumplirlas, además de los efectos positivos $y$ negativos que pueden generar en el feto, por ende se debería poner más énfasis en la suplementación que es una medida más fácil e idónea para cubrir estos requerimientos aumentados y de igual manera de estudiar sus beneficios en cuanto a la salud del bebe en formación.

Los resultados obtenidos en esta revisión sistemática tienen la aplicación práctica de sustentar la toma de decisiones para la indicación de suplementos nutricionales de colina, ácido fólico y DHA en mujeres embarazadas, ya que aporta evidencias de sus beneficios a nivel del desarrollo cognitivo de la descendencia especialmente en edades tempranas. Sin embargo, al haberse encontrado también un porcentaje de estudios con resultados contradictorios se recomienda su interpretación cautelosa y que dicha suplementación se realice tras un adecuado análisis nutricional previo.

\section{Limitaciones}

Como limitaciones de la presente revisión cabe destacar: i) el pequeño tamaño muestral de estudios hasta la fecha, por lo que se ve la necesidad de indagar más en el tema sobre todo en gestantes sanas (se observó la existencia de mayor número de estudios realizados en mujeres embarazadas con patologías, las cuales no fueron incluidas en esta revisión). ii) Se observó una alta heterogeneidad entre estudios, con una amplia diversidad de edades y cantidad suplementada de nutrientes. iii) Por último, es importante mencionar que esta es una revisión centrada de dimensiones cualitativas de neurodesarrollo (a través de cuestionarios y test cognitivos) y no se muestran variables que pudieran analizar los efectos a nivel cuantitativo (por ejemplo, a través de técnicas de neuroimagen) lo cual permitiría tener resultados más objetivables.

\section{CONCLUSIÓN}

$\mathrm{S}$ e identificó un efecto positivo de la alimentación dosificada cumpliendo con los requerimientos óptimos de la gestante principalmente en colina, ácido fólico, DHA y EPA, para el mayor desarrollo cognitivo del niño.

Se identificó a la colina como un nutriente esencial en la dieta prenatal, pero poco conocido, que realiza contribuciones importantes en el desarrollo del cerebro y la memoria visual. Se debe suplementar al menos $900 \mathrm{mg}$ /día en la dieta materna para que tenga un efecto positivo en la señalización durante la producción de células del sistema nervioso central para favorecer al aprendizaje del niño.

Se determinó que es necesario la suplementación de la gestante con 400 a $600 \mathrm{mg} /$ día de ácidos grasos poliinsaturados como el DHA y $150 \mathrm{mg}$ de EPA para que beneficie la formación del 
cerebro del niño y tenga efecto positivo especialmente en la atención visual.

Se estableció que se debe suplementar con ácido fólico la ingesta en la etapa de gestación con 400 a $600 \mu \mathrm{g} /$ día para ayudar al crecimiento de las neuronas y la síntesis de neurotransmisores, beneficiando el nivel cognitivo del niño y mejorando el razonamiento de palabras.

\section{REFERENCIAS BIBLIOGRÁFICAS}

1. Borge TC, Aase H, Brantsæter AL, Biele G. The importance of maternal diet quality during pregnancy on cognitive and behavioural outcomes in children: a systematic review and meta-analysis. BMJ Open. 2017;7(9):e016777. Disponible en: https://doi.org/10.1136/bmjopen-2017016777

2. National Institute of Child Health and Human Development (NICHD). Información sobre el embarazo. [Internet] 2020 [citado el 21 de mayo de 2021]. Disponible en: https://espanol.nichd.nih.gov/salud/temas/ pregnancy/informacion

3. Zeng Z, Liu F, Li S. Metabolic adaptations in pregnancy: A review. Ann Nutr Metab. 2017;70(1):59-65. Disponible en: https://doi.org/10.1159/000459633

4. Houwen S, Visser L, van der Putten A, Vlaskamp C. The interrelationships between motor, cognitive, and language development in children with and without intellectual and developmental disabilities. Res Dev Disabil. 2016;53-54:19-31. Disponible en: https://doi.org/10.1016/j.ridd.2016.01.012

5. Perichart-Perera 0 , Rodríguez-Cano AM, Gutiérrez-Castrellón P. Importancia de la suplementación en el embarazo: papel de la suplementación con hierro, ácido fólico, calcio, vitamina D y multivitamínicos. Gac Med Mex. 2020;156(Supl 3):S1-26.. Disponible en: https://doi.org/10.24875/ gmm.m20000434
6. Cox JT, Phelan ST. Nutrition during pregnancy. Obstet Gynecol Clin North Am. 2008;35(3):369-83, viii. Disponible en: https://doi.org/10.1016/j.ogc.2008.04.001

7. Espuig R, Noreña AL, Cortés E, GonzálezSanz J. Percepción de embarazadas y matronas acerca de los consejos nutricionales durante la gestación. Nutr Hosp. 2016;33(5):587. Disponible en: https://dx.doi.org/10.20960/nh.587

8. Crivellenti LC, Zuccolotto DCC, Sartorelli DS. Development of a Diet Quality Index Adapted for Pregnant Women. Rev Saude Publica. 2018; 52:59. Disponible en: https://doi.org/10.11606/s15188787.2018052000184

9. Institute of Medicine. Dietary Reference Intakes: The Essential Guide to Nutrient Requirements. Washington, DC: The National Academies Press. [Internet] 2016 [citado el 21 de mayo de 2021]. Disponible en: https://doi.org/10.17226/11537

10. Trumbo $P$, Schlicker $S$, Yates AA, Poos $M$, Food and Nutrition Board of the Institute of Medicine, The National Academies. Dietary reference intakes for energy, carbohydrate, fiber, fat, fatty acids, cholesterol, protein and amino acids. J Am Diet Assoc. 2002;102(11):1621-30. Disponible en: https://doi.org/10.1016/s00028223(02)90346-9

11. Kominiarek MA, Rajan P. Nutrition recommendations in pregnancy and lactation. Med Clin North Am. 2016;100(6):1199-215. Disponible en: https://doi.org/10.1016/ j.mcna.2016.06.004

12. Barretto L, Mackinnon MJ, Poy MS, Wiedemann A, López LB. Estado actual del conocimiento sobre el cuidado nutricional de la mujer embarazada. Rev Esp Nutr Hum Diet. 31 de octubre de 2014; 18(4):226-37. Disponible en: https://renhyd.org/index. php/renhyd/article/view/113

13. Sánchez, A. Guía de alimentación para embarazadas. Medicadiet. [Internet] 2015 [citado el 21 de mayo de 2021]. Disponible en: 
https://seedo.es/images/site/Guia_Aliment acion_Embazaradas_Medicadiet.pdf

14. Del Pino M, Nieto R, Meritano J, Rabosto Moleon R, Orden AB, Villafañe L, et al. Arch Argent Pediatr. 2020;118(5):S142. Disponible en: https://doi.org/10.5546/aap. 2020.s142

15. Firk C, Konrad K, Herpertz-Dahlmann B, Scharke W, Dahmen B. Cognitive development in children of adolescent mothers: The impact of socioeconomic risk and maternal sensitivity. Infant Behav Dev. 2018;50:238-46. Disponible en: https://doi.org/10.1016/j.infbeh.2018.02.0 02

16. Black MM. Impact of nutrition on growth, brain, and cognition. Nestle Nutr Inst Workshop Ser. 2018;89:185-95. Disponible en: https://doi.org/10.1159/000486502

17. Rombaldi Bernardi J, de Souza Escobar R, Ferreira CF, Pelufo Silveira P. Fetal and neonatal levels of omega-3: effects on neurodevelopment, nutrition, and growth. Scientific World Journal. 2012;2012:202473. Disponible https://doi.org/10.1100/2012/202473

18. Hurtado JA. Efecto de la suplementación materna con dha durante la gestación y lactancia sobre el desarrollo cognitivo/motor y visual del recién nacido en su primer año de vida. Tesis Doctoral, Universidad de Granada. [Internet] 2015

[citado el 21 de mayo de 2021]. Disponible en:

https://hera.ugr.es/tesisugr/2596303x.pdf

19. López R. Influencia de suplementar la dieta de la mujer gestante con DHA sobre el desarrollo cognitivo del niño. Trabajo de fin de Máster, Universitat de les Illes Balears. [Internet] 2016 [citado el 21 de mayo de 2021]. Disponible en: https://dspace.uib.es/xmlui/handle/11201 $/ 145930$

20. Villalobos M, Tous M, Canals J, Arija V. Vitamin D during pregnancy and neurodevelopment of the child: systematic review. An psicol. 2019;35(3):389-96.
Disponible en: https://dx.doi.org/10.6018/ analesps.35.3.326411

21. Rogne $T$, Tielemans MJ, Chong MF-F, Yajnik CS, Krishnaveni GV, Poston L, et al. Associations of maternal vitamin B12 concentration in pregnancy with the risks of preterm birth and low birth weight: A systematic review and meta-analysis of individual participant data. Am J Epidemiol. 2017;185(3):212-223. Disponible en: https://doi.org/10.1093/aje/kww212

22. Taylor R, Fealy S, Bisquera A, Smith R, Collins C, Evans T-J, et al. Effects of nutritional interventions during pregnancy on infant and child cognitive outcomes: A systematic review and meta-analysis. Nutrients. 2017;9(11):1265. Disponible en: https://doi.org/10.3390/nu9111265

23. Englund-Ögge L, Brantsæter AL, Juodakis J, Haugen M, Meltzer HM, Jacobsson B, et al. Associations between maternal dietary patterns and infant birth weight, small and large for gestational age in the Norwegian Mother and Child Cohort Study. Eur J Clin Nutr. 2019;73(9):1270-82. Disponible en: https://doi.org/10.1038/s41430-018-0356$\mathrm{y}$

24. Liberati A, Altman DG, Tetzlaff J, Mulrow C, Gøtzsche PC, Ioannidis JPA, et al. The PRISMA statement for reporting systematic reviews and meta-analyses of studies that evaluate health care interventions: explanation and elaboration. J Clin Epidemiol. 2009;62(10):e1-34.Disponible en: https://doi.org/10.1016/j.jclinepi.2009. 06.006

25. Higgins JPT, Thomas J, Chandler J, Cumpston M, Li T, Page MJ, et al. Cochrane Handbook for Systematic Reviews of Interventions version 6.2 (updated February 2021). Cochrane. [Internet] 2021 [citado el 21 de mayo de 2021]. Disponible en: www.training.cochrane.org/handbook

26. Caudill MA, Strupp BJ, Muscalu L, Nevins JEH, Canfield RL. Maternal choline supplementation during the third trimester of pregnancy improves infant information processing speed: a randomized, double- 
blind, controlled feeding study. FASEB J. 2018;32(4):2172-80. Disponible en: https://doi.org/10.1096/fj.201700692rr

27. McNulty $H$, Rollins $M$, Cassidy $T$, Caffrey A, Marshall B, Dornan J, et al. Effect of continued folic acid supplementation beyond the first trimester of pregnancy on cognitive performance in the child: a follow-up study from a randomized controlled trial (FASSTT Offspring Trial). BMC Med. 2019;17(1):196. Disponible en: https://doi.org/10.1186/s12916-0191432-4

28. Colombo J, Shaddy DJ, Gustafson K, Gajewski BJ, Thodosoff JM, Kerling E, et al. The Kansas University DHA Outcomes Study (KUDOS) clinical trial: long-term behavioral follow-up of the effects of prenatal DHA supplementation. Am J Clin Nutr. 2019;109(5):1380-92. Disponible en: https://doi.org/10.1093/ajcn/nqz018

29. Ramakrishnan U, Gonzalez-Casanova I, Schnaas L, DiGirolamo A, Quezada AD, Pallo $\mathrm{BC}$, et al. Prenatal supplementation with DHA improves attention at $5 \mathrm{y}$ of age: a randomized controlled trial. Am J Clin Nutr. 2016;104(4):1075-82. Disponible en: https://doi.org/10.3945/ajcn.114.101071

30. Azaryah H, Verdejo-Román J, MartinPérez C, García-Santos JA, Martínez-Zaldívar C, Torres-Espínola FJ, et al. Effects of maternal fish oil and/or 5-methyltetrahydrofolate supplementation during pregnancy on offspring brain resting-state at 10 years old: A follow-up study from the NUHEAL randomized controlled trial. Nutrients. 2020;12(9):2701. Disponible en: https://doi.org/10.3390/nu12092701

31. Colombo J, Gustafson KM, Gajewski BJ, Shaddy DJ, Kerling EH, Thodosoff JM, et al. Prenatal DHA supplementation and infant attention. Pediatr Res. 2016;80(5):656-62. Disponible https://doi.org/10.1038/pr.2016.134

32. Mulder KA, King DJ, Innis SM. Omega-3 fatty acid deficiency in infants before birth identified using a randomized trial of maternal DHA supplementation in pregnancy. PloS one. 2014; 9(1), e83764. Disponible en: https://doi.org/10.1371/ journal.pone.008376

33. Gould JF, Makrides M, Colombo J, Smithers LG. Randomized controlled trial of maternal omega-3 long-chain PUFA supplementation during pregnancy and early childhood development of attention, working memory, and inhibitory control. Am J Clin Nutr. 2014;99(4):851-9. Disponible en: $\quad$ https://doi.org/10.3945/ajcn.113. 069203

34. Meldrum S, Dunstan JA, Foster JK, Simmer K, Prescott SL. Maternal fish oil supplementation in pregnancy: a 12 year follow-up of a randomised controlled trial. Nutrients. 2015;7(3):2061-7. Disponible en: https://doi.org/10.3390/nu7032061

35. Rees A, Sirois $S$, Wearden A. Prenatal maternal docosahexaenoic acid intake and infant information processing at $4.5 \mathrm{mo}$ and 9mo: A longitudinal study. PLoS One. 2019;14(2):e0210984. Disponible en: https://doi.org/10.1371/journal.pone.0210 984

36. Prado EL, Dewey KG. Nutrition and brain development in early life. Nutr Rev. 2014;72(4):267-84. Disponible en: https://doi.org/10.1111/nure.12102

37. Wallace $T$, Fulgoni V. Usual choline intakes are associated with egg and protein food consumption in the United States. Nutrients. 2017;9(8):839. Disponible en: https://doi.org/10.3390/nu9080839

38. Korsmo HW, Jiang X, Caudill MA. Choline: Exploring the growing science on its benefits for moms and babies. Nutrients. 2019;11(8):1823. Disponible en: https://doi.org/10.3390/nu11081823

39. Boeke CE, Gillman MW, Hughes MD, Rifas-Shiman SL, Villamor E, Oken E. Choline intake during pregnancy and child cognition at age 7 years. Am J Epidemiol. 2013;177(12):1338-47. Disponible en: https://doi.org/10.1093/aje/kws395

40. Bahnfleth C, Canfield R, Nevins J, Caudill M, Strupp B. Prenatal choline 
supplementation improves child colorlocation memory task performance at $7 \mathrm{Y}$ of age (FS05-01-19). Curr Dev Nutr. 2019; 3(Supplement_1). Disponible en: https://doi.org/10.1093/cdn/nzz048.fs0501-19

41. Ross RG, Hunter SK, McCarthy L, Beuler J, Hutchison AK, Wagner BD, et al. Perinatal choline effects on neonatal pathophysiology related to later schizophrenia risk. Am J Psychiatry. 2013;170(3):290-8. Disponible en: $\quad$ https://doi.org/10.1176/appi.ajp. 2012.12070940

42. Ross RG, Hunter SK, Hoffman MC, McCarthy L, Chambers BM, Law AJ, et al. Perinatal phosphatidylcholine supplementation and early childhood behavior problems: Evidence for CHRNA7 moderation. Am J Psychiatry. 2016;173(5):509-16. Disponible en: https://doi.org/10.1176/appi.ajp.2015.150 91188

43. Devarshi PP, Grant RW, Ikonte CJ, Hazels Mitmesser S. Maternal omega-3 nutrition, placental transfer and fetal brain development in gestational diabetes and preeclampsia. Nutrients. 2019;11(5):1107. Disponible en: https://doi.org/10.3390/ nu11051107

44. Zhang Z, Fulgoni V III, Kris-Etherton P, Mitmesser S. Dietary intakes of EPA and DHA omega-3 fatty acids among US childbearingage and pregnant women: An analysis of NHANES 2001-2014. Nutrients. 2018;10(4):416. Disponible en: https://doi.org/10.3390/nu10040416

45. Hibbeln JR, Davis JM, Steer C, Emmett P, Rogers I, Williams C, et al. Maternal seafood consumption in pregnancy and neurodevelopmental outcomes in childhood (ALSPAC study): an observational cohort study. Lancet. 2007;369(9561):578-85. Disponible en: https://doi.org/10.1016 /s0140-6736(07)60277-3

46. Dunstan JA, Simmer K, Dixon G, Prescott SL. Cognitive assessment of children at age
$2(1 / 2)$ years after maternal fish oil supplementation in pregnancy: a randomised controlled trial. Arch Dis Child Fetal Neonatal Ed. 2008;93(1):F45-50. Disponible en: https://doi.org/10.1136/adc. 2006.099085

47. Judge MP, Harel O, Lammi-Keefe CJ. Maternal consumption of a docosahexaenoic acid-containing functional food during pregnancy: benefit for infant performance on problem-solving but not on recognition memory tasks at age 9 mo. Am J Clin Nutr. 2007;85(6):1572-7. Disponible en: https://doi.org/10.1093/ajcn/85.6.1572

48. Castaño E, Piñuñuri R, Hirsch $S$, Ronco AM. Folatos y Embarazo, conceptos actuales: ¿Es necesaria una suplementación con Ácido Fólico? Rev Chil Pediatr. 2017;88(2):199$206 . \quad$ Disponible en: https://doi.org/10.4067/s037041062017000200001

49. Chatzi L, Papadopoulou E, Koutra K, Roumeliotaki T, Georgiou V, Stratakis N, et al. Effect of high doses of folic acid supplementation in early pregnancy on child neurodevelopment at 18 months of age: the mother-child cohort "Rhea" study in Crete, Greece. Public Health Nutr. 2012;15(9):1728-36. Disponible en: https://doi.org/10.1017/s1368980012000 067

51. Villamor E, Rifas-Shiman SL, Gillman MW, Oken E. Maternal intake of methyldonor nutrients and child cognition at 3 years of age: Maternal diet and childhood cognition. Paediatr Perinat Epidemiol. 2012;26(4):328-35. Disponible en: https://doi.org/10.1111/j.1365-

3016.2012.01264.x

Conflicto de interese: Los autores declaran que no existe conflicto de intereses para la publicación del presente artículo.

Financiamiento: Autofinanciamiento

Agradecimiento: No declaran 